INTERPLANETARY DUST :

ZODIACAL LIGHT AND OPTICAL STUDIES 


\title{
THE ZODIACAL CLOUD COMPLEX
}

\author{
A.C. LEVASSEUR-REGOURD and J.B. RENARD \\ Université Paris-6/Aéronomie - BP 3 \\ 91371 Verrieres le Buisson France \\ R. DUMONT \\ Observatoire de Bordeaux \\ 33270 Floirac France
}

\begin{abstract}
The physical properties of the interplanetary dust grains are, out of the ecliptic plane, mainly derived from observations of zodiacal light in the visual or infrared domains. The bulk optical properties (polarization, albedo) of the grains are demonstrated to depend upon their distance to the Sun (at least in a $0.1 \mathrm{AU}$ to $1.7 \mathrm{AU}$ range in the symmetry plane) and upon the inclination of their orbits (at least up to $22^{\circ}$ ). Classical models assuming the homogeneity of the zodiacal cloud are no longer acceptable. A hybrid model, with a mixture of two populations, is proposed. It suggests that various sources (periodic comets, asteroids, non periodic comets...) play an important role in the replenishment of the zodiacal cloud complex.
\end{abstract}

\section{Introduction}

The tridimensional distribution of interplanetary dust grains number densities and physical properties can be, as perfectly summarized a few years ago by the late R.H. Giese (1986), obtained by two methods. "The first method implies direct measurements and analysis of particles impacts on spaceborne detectors... The disadvantages are selection effects and the limited number of impact events... The second method is based on sunlight scattered by interplanetary dust grains... A huge number of particles is involved, which allows to derive the 3D-distribution without statistical problems. However... local number densities can only be derived indirectly i.e. by model computations using some simplifying assumptions, except in a few special cases...".

Interplanetary dust grains have indeed been collected in the Earth's environment (deep sea sediments, polar ices, stratosphere, Earth's orbit with LDEF or HITEN, see for instance McDonnell, 1991 or Igenbergs et al., 1991). Also their impacts on the Moon or on various spacecraft (e.g. Galileo, Grün et al., 1991) have been studied. The chemical composition of the dust grains deduced by analysis of the previous samples supports both the comets or the carbonaceous chondrites origin hypothesis.

However, due to the limited number of impact events (which, anyhow, are up to now restricted to the ecliptic plane), the properties (e.g. density, size, polarization, albedo) of the dust grains have been mainly derived from observations of light scattered or emitted by interplanetary dust. At $1 \mathrm{AU}$ from the Sun, and up to solar distances smaller than $2.5 \mathrm{AU}$ in the ecliptic plane, the so-called zodiacal light is indeed a significant contribution to the light of the night sky, both in the visual domain (scattered solar light) and in the infrared domain (thermal emission).

Intensity and polarization of scattered light have been measured by various groups. A smoothed table of intensities (Levasseur-Regourd and Dumont, "LRD", 1980) and an extensive compilation of intensities and polarizations (Fechtig-Leinert-Grün, "FLG", 1981 ) are available. Thermal emission from interplanetary dust has been measured from

A.C. Levasseur-Regourd and H. Hasegawa (eds.), Origin and Evolution of Interplanetary Dust, 131-138. (c) 1991 Kluwer Academic Publishers, Printed in Japan. 
balloons, rockets and IRAS spacecraft, mainly between $60^{\circ}$ and $120^{\circ}$ solar elongation. Local bulk physical properties, together with some informations about the shape of the cloud, can therefore be derived by model fittings or inversion techniques.

\section{Models of the zodiacal dust cloud}

\subsection{CLASSICAL ASSUMPTIONS}

As shown by Dumont (1973), the difference between two consecutive observations made tangentially to the direction of motion (of the Earth or of a moving probe) provides the elemental contribution of the section of the line of sight where the observer is located. For any other direction of observation and section of the line of sight, various assumptions are required.

The zodiacal cloud has been shown to have a warped symmetry surface near the ecliptic plane (Misconi, 1980). From both visual and thermal observations, its parameters have been computed to be of the order of $i=1.5^{\circ}, \Omega=90^{\circ}$ near the Earth's orbit (Dumont and Levasseur-Regourd, 1978, 1987; Hauser et al., 1984; Murdock and Price, 1985). The rotational symmetry assumption with respect to this so-called symmetry plane is fairly acceptable.

It is also generally assumed that the zodiacal cloud is relatively smooth. Local heterogeneities, due to dust from cometary or asteroidal origin, have indeed been suspected in the visual data (Levasseur and Blamont, 1976), and found in the thermal data (Sykes et al., 1986 ; Dermott et al., 1986). They are, however, mainly restricted to cometary orbits and to the asteroid belt and can therefore (at least to the first order) be neglected.

A third assumption is the homogeneity assumption, i.e. the assumption that the physical properties of the grains are the same all over the solar system. With such an hypothesis, the radial dependence of the dust distribution is immediately derived, in the symmetry plane, from that of the intensity. It would increase approximately as $\mathrm{R}^{-1.25}$ with decreasing solar distance R (Dumont and Sanchez, 1975 ; Leinert et al., 1977).

\subsection{TRIDIMENSIONAL MODELS}

With the smoothness assumption, analytical representations of the 3D distribution of interplanetary dust can be derived. As carefully reviewed by Giese et al. $(1986,1989)$ and Kneissel (1991), several models are available. With the rotational symmetry assumption, isodensity lines (leading to figurative models names) can be drawn in the helioecliptic meridian plane. It has to be emphasized that an homogeneity assumption is almost always made with a density power law $R^{-n}$ ( $n$ in a 1 to 1.3 range) in the symmetry plane.

The most simple models developed to fit the observations have been the ellipsoid or blown up ellipsoid models (Giese and v. Dziembovski, 1969 ; Dumont, 1976a). Also widely used have been the fan or flattened fan models which show a depression towards the Sun (Leinert et al., 1976, 1981).

A puzzling result is that the visual observations seem to be better fitted by a bulge than by a depression towards the Sun, with the opposite result for the infrared observations. Various bulge models have been developed, e.g. the sombrero (Dumont, 1976b), cosine (Giese et al., 1985), revised cosine (Rittich, 1986) or modified fan (Lumme and Bowell, 1985) models. Also depression models have been developed, by Murdock and Price (1985), Good et al. (1986) and Lamy and Perrin (1986). 
It is likely that such fundamental discrepancies are due to the fact that most of the models assume the physical properties of the grains to be the same everywhere in the solar system. Results obtained both from Helios 1,2 and Pioneer 10,11 space probes suggest that this is not the case (Leinert et al., 1981; Toller and Weinberg, 1985). Attempts to some inversion of the observations do confirm that the dust cloud is far from being homogeneous.

\subsection{NODES OF LESSER UNCERTAINTY METHOD}

Only assuming the existence of a symmetry plane and the relative smoothness of the cloud (together with the absence of dust at infinity), some inversions are feasible both in the symmetry plane, and in the plane perpendicular to the Sun-Earth line at 1 AU from the Sun, socalled the tangential plane (Fig.1).

It is found that the mathematical smooth functions (polynomial, exponential, lorentzian, Fourier series) which could represent the local brightnesses (integrals equal to the measured brightnesses, asymptotical decrease to zero with increasing solar distance) have to focus in some nodal regions where the local contributions are derived with less uncertainty than elsewhere.

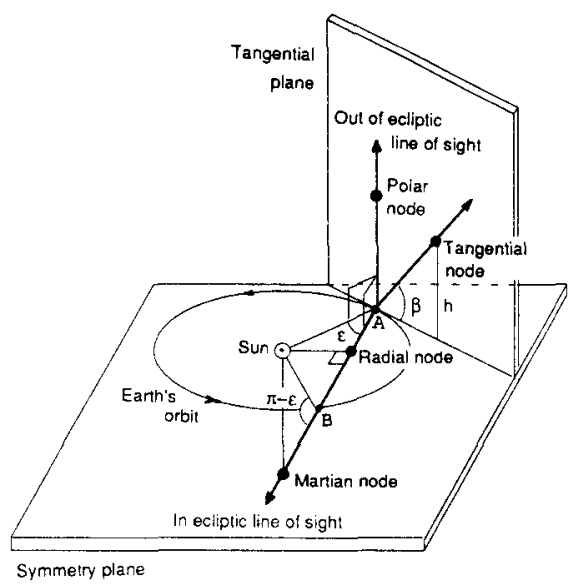

Figure 1 - Geometry of zodiacal observations

In the symmetry plane (Dumont, 1983; Dumont and Levasseur-Regourd, 1985a), two nodes can be found once integrated brightnesses have been measured from $A$ at elongation $\varepsilon$ and from $B$ at elongation $180^{\circ}-\varepsilon$. At the radial node, the phase angle is equal to $90^{\circ}$ and the solar distance (in AU) is equal to sin $\varepsilon$. At the martian node, the solar distance $\mathrm{R}$ is about $1.54 \mathrm{AU}$ and the phase angle is equal to $\sin ^{-1}[(\sin \varepsilon) / \mathrm{R}]$.

In the tangential plane, one node is obtained once local brightnesses at $1 \mathrm{AU}$ have been derived from inversion in the symmetry plane, and once integrated brightnesses at ecliptic latitude $\beta$ are available. The elevation upon the symmetry plane of the tangential (possibly polar) node, equal to $\sin \beta / \operatorname{tg} \alpha$, where $\alpha$ is the phase angle is found to remain smaller than 0.4 AU. A large fraction of the zodiacal cloud can therefore be locally probed and the evolution of the local properties of the grains can be studied all over this domain.

\section{New results on interplanetary dust properties}

\subsection{LOCAL INTENSITY}

2.1.1. Radial dependence in the symmetry plane. The local intensities are immediately obtained at a phase angle $\alpha=90^{\circ}$ from results derived at the radial nodes between $\mathrm{R}=0.09 \mathrm{AU}$ and $1 \mathrm{AU}$. There is an excellent agreement between the two sets of data, from Leinert et al. (1976) observations in the inner solar system, and from LRD (1980) smoothing for solar elongations greater than $30^{\circ}$. The error bars (due to the method of inversion) are significantly below $10 \%$. Also, the local intensities at $\alpha=90^{\circ}$ are extrapolated between $1 \mathrm{AU}$ and $1.74 \mathrm{AU}$ from results derived in the symmetry plane at the tangential nodes. 
There is a fair agreement between the local values of the intensity and the sum of their two polarized components (as derived from Dumont and Sanchez, 1975 or from FLG, 1981). From the values presented in Fig. 2, the local intensity is found to vary as

$\mathrm{R}^{-1.24}\left(0.999\right.$ correlation). We therefore suggest that the local intensity (in $\mathrm{S}_{10}(\mathrm{~V}) / \mathrm{rad}$ ) can be described by a $180 \times \mathrm{R}^{-(1.25 \pm 0.05)}$ power law. It is of interest to notice that such a result would perfectly agree with the density power law previously mentioned if the local cross section and albedo were assumed to be constant.

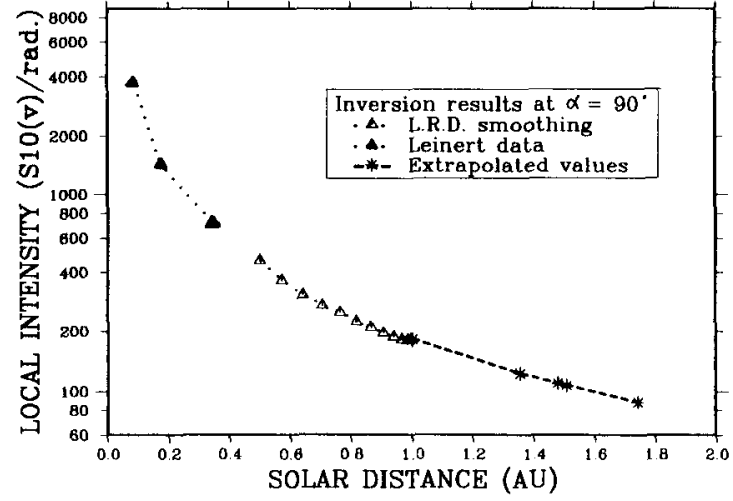

Figure 2 - Radial dependence of the local intensity at $90^{\circ}$

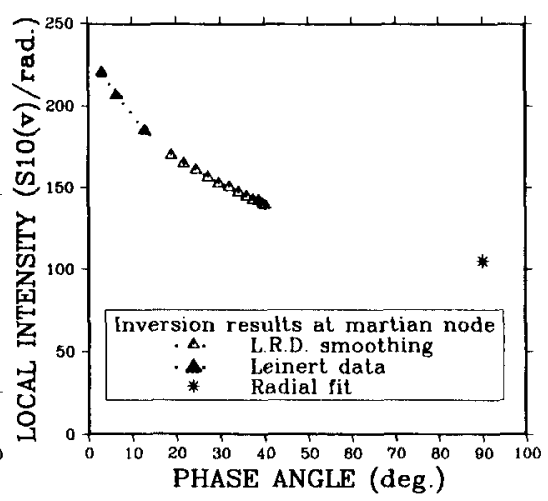

Figure 3 - Phase dependence of the local intensity at $1.5 \mathrm{AU}$

2.1.2. Phase dependence in the symmetry plane. The local intensities are immediately obtained at a solar distance of about 1.5 AU from results derived at martian nodes between $\alpha=3^{\circ}$ and $40^{\circ}$ (whence the extrapolation mentioned in 2.1.1). The intensity extrapolated at $\alpha=90^{\circ}$ agrees well with the intensity extrapolated at $1.5 \mathrm{AU}$. The relative error bars remain smaller than $1 \%$.

The decrease in intensity with increasing phase angle (backscattering) is of the order of $0.015 \pm 0.005$ magnitude per degree, with a slight opposition effect below $7^{\circ}$ (Fig. 3). Such a phase function is somewhat reminiscent of the phase function obtained for cometary dust (Meech and Jewitt, 1987), but it is likely that the backscattering function is less steep than in the cometary case.

2.1.3. Elevation dependence above the symmetry plane. From results obtained in the tangential plane, the local intensities at constant solar distance $(\approx 1.5 \mathrm{AU})$ are derived up to an elevation $h$ of about $0.3 \mathrm{AU}$ above the symmetry plane. The relative error bars remain smaller than $2^{\circ}$.

The significant decrease of intensity with increasing elevation (Fig. 4) illustrates the flattening of the dust cloud. It should be noticed that the curvature of the graph is definitely in better agreement with a solar bulge model than with a solar depression model. The best fit is obtained for the cosine models.

\subsection{POLARIZATION}

2.2.1. Radial dependence in the symmetry plane. The local polarizations at constant phase angle $\left(90^{\circ}\right)$ are also obtained between $\mathrm{R}=0.09 \mathrm{AU}$ and $1 \mathrm{AU}$ from results at the radial nodes. The discrepancy between the two sets of values is due to the fact that Dumont and 
demonstrated in this domain. Such a result illustrates the heterogeneity of the dust cloud in the tangential plane, and suggests that the properties of the grains depend upon the inclination of their orbits.

\subsection{ALBEDO}

2.3.1. Radial dependence in the symmetry plane. Inversion of the integrated thermal intensities, as measured by IRAS spacecraft (Hauser et al., 1984) or ZIP rocket (Murdock and Price, 1985) at two wavelengths, allows to estimate local temperatures in the symmetry plane (Levasseur-Regourd and Dumont, 1987; Dumont and LevasseurRegourd, 1988). From results obtained at the radial and tangential nodes, values can be derived between approximately $0.5 \mathrm{AU}$ and $1.7 \mathrm{AU}$. The absolute value of the local temperature at $1 \mathrm{AU}$ is of about $255 \mathrm{~K}$ from IRAS observations, and of about $295 \mathrm{~K}$ from ZIP observations. However, the two sets of data provide the same $R^{-0.35}$ power law ( 0.990 correlation) for the decrease of temperature with increasing solar distance. The deviation of the temperature gradient from - 0.50 is likely to come from distinct heliocentric changes in the visible and infrared cross sections.

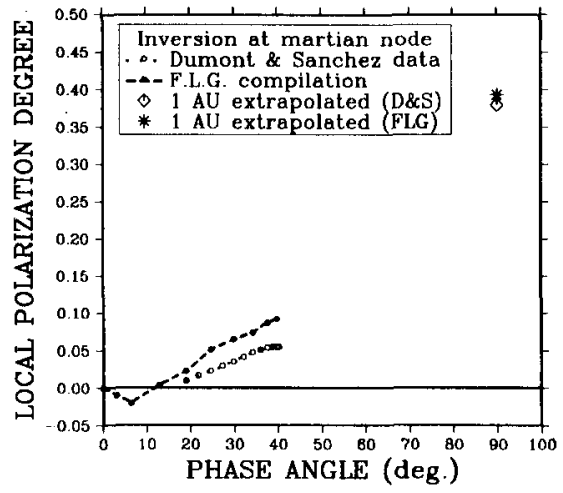

Figure 6 - Phase dependence of the polarization degree

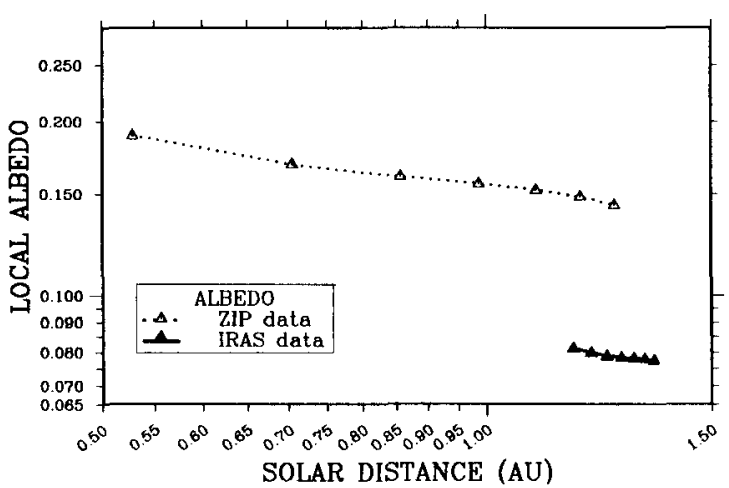

Figure 7 - Radial dependence of the local albedo at $90^{\circ}$

The local bulk albedo at $\alpha=90^{\circ}$ (by extension of the definition of the geometric albedo, Hanner et al, 1981) is estimated from results obtained for local intensity (energy scattered in the visual domain) and for local temperature (thermal energy reemitted in the infrared). As can be seen on Fig. 7, the absolute values of the albedo depend upon the calibration of the thermal observations. They are, however, quite low (typically of the order of 0.08 at 1 AU from IRAS data). The trend to an increase with decreasing solar distance is nevertheless fairly well established from $0.5 \mathrm{AU}$ to $1.5 \mathrm{AU}$, in agreement with previous results derived from other techniques (Fechtig, 1984; Lumme and Bowell, 1985).

The gradient of a power law fitting these local bulk albedos is computed to be of the order of $-0.30 \pm 0.10$. Since, as shown by Giese and Kinateder (1986), a scattering function gradient $-\mathrm{n}_{1}$ and an albedo gradient $-\mathrm{n}_{2}$ lead to a density gradient $-\mathrm{n}=-\left(\mathrm{n}_{1}\right.$ $\mathrm{n}_{2}$ ), it can be concluded that the density decreases as $\mathrm{R}-0.90 \pm 0.15$. Such a value is in perfect agreement with the expected density law (1/R) for interplanetary grains under Poynting-Robertson effect (Hanner, 1980). 
Sanchez (1975) integrated polarization values are a few percent smaller than those given in FLG (1981) compilation. The relative error bars are below $10 \%$.

The trend to a decrease with decreasing solar distance, suspected from the decrease in integrated polarizations in the inner solar system (Leinert et al., 1981), is confirmed (Dumont and Levasseur-Regourd, 1985b). In the 0.7 to $1 \mathrm{AU}$ range, the local polarization varies as $\mathrm{R}^{0.80}$. A very strong decrease seems to appear below 0.3 AU (Fig. 5). Such a result, based on observations at low solar elongations (Blackwell et al., 1967; Leinert et al., 1976) seems to be confirmed by inversion results obtained in the near coronal region (Dumont and Pelletanne, 1981 ; Mann, 1990). It could suggest a drastic change in the physical properties of the grains.

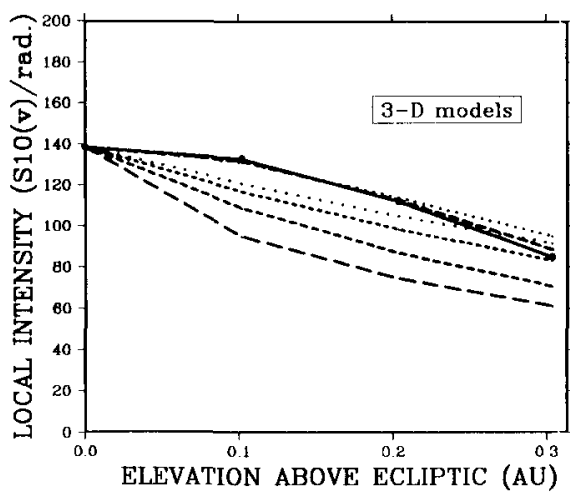

Figure 4 - Comparison of local intensity with computed intensities (dashed lines). From top to bottom, on the left side : ellipsoid, fan, cosine, (Good) infrared, modified fan and (Lamy) depression models

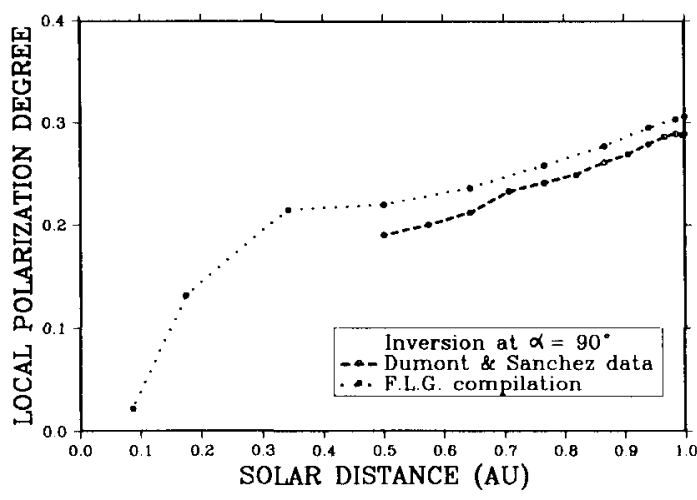

Figure 5 - Radial dependence of polarization at $90^{\circ}$

2.2.2. Phase dependence in the symmetry plane. The local polarizations at constant solar distance (of about $1.5 \mathrm{AU}$ ) are obtained between $3^{\circ}$ and $40^{\circ}$ from results at the martian nodes. The error bars are smaller than $2 \%$, except for the points at $90^{\circ}$ which are extrapolated from results at the radial nodes between 0.6 and $1 \mathrm{AU}$.

Since the integrated polarizations mesured from $\mathrm{A}$ at small elongations are uncertain, the local values at small phase angles may be disputable. However, the local polarization is likely to be negative (electric vector in the scattering plane, instead of being perpendicular to it) for $\alpha$ smaller than $10^{\circ}$, to change sign below $20^{\circ}$, and to increase almost linearly up to $40^{\circ}$ (Fig. 6). This result confirms the trends obtained from a different approach, using the few available observations in the Gegenschein region (Levasseur-Regourd et al., 1990). Again, the polarization function of interplanetary dust is reminiscent of the function obtained for comets. However, the smaller value of the phase angle at the neutral point (in a $10^{\circ}$ to $20^{\circ}$ range, instead of $21^{\circ} \pm 1^{\circ}$ for comet Halley's dust) could suggest that the interplanetary dust grains are not identical to the cometary ones.

2.2.3. Elevation dependence in the tangential plane. A map of the polarization degree at $\alpha=90^{\circ}$ is derived in the tangential plane from results obtained at the tangential and martian nodes in that plane, after normalization at constant phase angle (Renard et al. 1991, fig. 4). It extends up to $0.4 \mathrm{AU}$ above the Earth towards the pole and as far as 1.5 AU in the symmetry plane.

The polarization decrease (at constant phase angle and solar distance), previously noticed towards the pole of the symmetry plane (Levasseur-Regourd and Dumont, 1990), is 
2.3.2. Elevation dependence above the symmetry plane. The local bulk albedo at $1 \mathrm{AU}$ and $90^{\circ}$ is derived from local intensities and temperatures at the polar nodes of the Earth-pole line of sight, and found to increase with increasing elevation upon the symmetry plane (Renard et al., 1991, fig. 3).

Again, an increase in albedo is found to be correlated with a decrease in polarization. The evolution of albedo with increasing elevation confirms the result obtained for polarization, and suggests that the grains found on inclined orbits are quite different from those which are predominant in the symmetry plane.

\section{Conclusions}

To summarize, the heterogeneity of the zodiacal cloud is evident, both in the symmetry plane and in the plane perpendicular to the Sun-Earth line at 1 AU. In the symmetry plane, the local polarization decreases and the local albedo increases with decreasing solar distance. In the tangential plane, the local polarization decreases and the local albedo increases with increasing elevation, i.e. with increasing inclination of the grains orbits upon the symmetry plane. Besides, the slight discrepancies between the phase and polarization functions for comet Halley or interplanetary dust in the symmetry plane need to be emphasized, together with the discrepancies between the various models fitting visible or infrared observations towards the Sun.

The previous results suggest that the cloud is a mixture of two populations of grains, one (1) with low albedo and high polarization, the other (2) the reverse. The former (1) would be more flattened and slower in its radial decrease of density than the latter (2), which could be rather isotropic in its distribution. It is tempting to associate population 1 (to which infrared observations are quite sensitive) to dark grains which could originate in the processed crust of active periodic comets or extinct comets, and in asteroids. Also, population 2 (to which infrared observations are less sensitive) could be associated to new comets on randomly inclined orbits or to $\beta$ meteoroids. However, without discussing the question of the various origins of the interplanetary dust complex, it is now clear that the 3-D models which do not take into account any spatial change in the properties of the grains are not acceptable and that at least a bimodal population is required to describe the local properties of the zodiacal cloud.

\section{References}

Blackwell D.E., Dewhirst D.W., Ingham M.F. (1967), Adv. Astron. Astrophys. 5, 1-69.

Dermott S.F., Nicholson P.D., Wolven B. (1986), in C.I. Lagerkvist et al. (eds.), Asteroids, comets, meteors II, HSC, Uppsala, 583-594.

Dumont R. (1973), Planet. Space Sci., 21, 2149-2155.

Dumont R. (1976), in H. Elsässer and H. Fechtig (eds.), Interplanetary dust and zodiacal light, Springer-Verlag, Berlin, 85-100.

Dumont R. (1976), IAU General assembly.

Dumont R. (1983), Planet. Space Sci., 31, 1381-1387.

Dumont R. and Levasseur-Regourd A.C. (1978), Astron. Astrophys., 64, 9-16.

Dumont R. and Levasseur-Regourd A.C. (1985a), Planet. Space Sci., 33, 1-9.

Dumont R. and Levasseur-Regourd A.C. (1985b), in R.H. Giese and P. Lamy (eds.), Properties and interactions of interplanetary dust, D. Reidel Publishing co., Dordrecht, 207-213. 
Dumont R. and Levasseur-Regourd A.C. (1987), in Z. Ceplecha and P. Pecina (eds.), Interplanetary matter, Astron. Inst. of the Czechoslovak Acad. of Sciences, 67, 281-284.

Dumont R. and Levasseur-Regourd A.C. (1988), Astron. Astrophys., 191, 154-160.

Dumont R. and Pelletanne B. (1981), C.R. Acad. Sci. Paris, 293, II, 377-380.

Dumont R. and Sanchez F. (1975), Astron. Astrophys., 38, 405-412.

Fechtig H. (1984), Adv. Space Res., 4, 5-12.

Fechtig H., Leinert C., Grün E. (1981) "FLG", in K.Schaifers and H. Voigt (eds.), Landolt-Börnstein N.S., 2a, Springer-Verlag, Berlin, 228-243.

Giese R.H. and v. Dziembowski C. (1969), Planet. Space Sci., 17, 949-956.

Giese R.H., Kinateder G., Kneissel B., Rittich U. (1985), in R.H. Giese and P. Lamy (eds.) Properties and interactions of interplanetary dust, D. Reidel Publishing co. Dordrecht, 255-259.

Giese R.H. and Kinateder G. (1986), in R.G. Marsden (ed.), The Sun and the heliosphere in 3 dimensions, D. Reidel Publishing co., Dordrecht, 441-454.

Giese R.H., Kneissel B., Rittich U. (1986), Icarus, 68, 395-411.

Giese R.H. and Kneissel B. (1989), Icarus, 81, 369-378.

Good J.C., Hauser G.M., Gautier T.N. (1986), Adv. Space Res., 6, 83-86.

Grün E. et al. (1991), (this issue).

Hanner M.S. (1980), Icarus, 43, 373-380.

Hanner M.S. (1981), Astron. Astrophys., 104, 42-46.

Hauser M.G., Gillett F.C., Low F.L., Gautier T.N., Beichman C.A., Neugebauer G., Aumann H.H., Baud B., Boggess N. Emerson J.P., Houck J.R., Soifer B.T., Walker R.G. (1984), Astrophys. J., 278, L15-L18.

Igenbergs E. et al. (1991), (this issue).

Kneissel B. (1991), (this issue).

Lamy P. and Perrin J.M. (1986), Astron. Astrophys., 163, 269-286.

Leinert C., Link H., Pitz E., Giese R.H. (1976), Astron. Astrophys., 47, 221-230.

Leinert C., Pitz E., Hanner M., Link H. (1977), J. Geophys., 42, 669-704.

Leinert C., Richter I, Pitz E., Planck B. (1981), Astron. Astrophys., 103, 177-188.

Levasseur A.C. and Blamont J.E. (1976), in H. Elsässer and H. Fechtig (eds.) Interplanetary dust and zodiacal light, Springer-Verlag, Berlin, 58-62.

Levasseur-Regourd A.C. and Dumont R. (1980), "LRD", Astron. Astrophys. , 84, 277-279.

Levasseur-Regourd A.C. and Dumont R. (1987), Adv. Space Res., 6, 7, 87-90.

Levasseur-Regourd A.C. and Dumont R. (1990), Adv. Space Res., 10, 3, 163-170.

Levasseur-Regourd A.C., Dumont R., Renard J.B. (1990), Icarus, 86, 264-272.

Lumme K. and Bowell E. (1985), Icarus, 62, 54-71.

McDonnell J.A.M. (1991), (this issue).

Mann I. (1990), Dissertation, Ruhr Universität, Bochum.

Meech K.J. and Jewitt D.C. (1987), Astron. Astrophys., 187, 585-593.

Misconi N.Y. (1980), in I. Halliday and B.A. McIntosh (eds.), Solid particles in the solar system, R. Reidel Publishing co., Dordrecht, 49-53.

Murdock T.L. and Price D. (1985), Astron. J. 90, 375-386.

Renard J.B., Levasseur-Regourd A.C., Dumont R. (1991), (this issue).

Rittich U. (1986), Diplomarbeit, Ruhr-Universität, Bochum.

Sykes M.V., Lebofsky L.A., Hunten D.M., Low F.J. (1986), Science, 232, 1115-1117.

Toller G.N. and Weinberg J.L. (1985), in R.H. Giese and P. Lamy (eds.) Properties and interactions of interplanetary dust, D. Reidel Publishing co., Dordrecht, 21-25. 